\title{
INTERDISCIPLINARY RESEARCH ON IMPAIRED MOBILITY, DISABILITY AND REHABILITATION IN SELANGOR
}

\author{
Manderson L', Zaliha $\mathrm{O}^{2}$, Rameezan $B A R^{2}$, Nooreini $\mathrm{AH}^{2}$, Soh $\mathrm{SB}^{2}$ and Disler $\mathrm{P}^{3}$ \\ School of Psychology, Psychiatry and Psychological Medicine, Monash University, 3800 Australia \\ 2 Department of Allied Health Sciences, Faculty of Medicine, University of Malaya, 50603 Kuala Lumpur, Malaysia \\ 3 School of Rural Health, Monash University, 3800 Australia
}

\begin{abstract}
Demographic, economic and social changes have had major impact on health and illness globally, including in Malaysia, and present significant challenges to the structure and delivery of health services. While these changes have influenced the epidemiology of disease, the diagnosis, experience and response to changes in health status for individuals and their families are influenced by additional environmental and personal factors. We describe these factors in relation to our ongoing research program on personal and social aspects of impairment and disability. The Resilience study aims to understand how people with impairments and their families live with chronic health conditions, how these conditions impact on self-esteem, social relationships and societal participation, and how structure, context and environment affect individual functioning, disability and well-being. We described our methodology and summarize the baseline data that will inform our future enquiries. (JUMMEC 2006; 9(2): 12-17)
\end{abstract}

KEYWORDS: Disabled persons, locomotor activity, Malaysia, quality of life, social impact

\section{Introduction}

The global increase in the prevalence of chronic diseases in middle and low as well as high income countries reflects both the success of public health interventions and improvements in the treatment of disease, and social changes related to industrialization, urbanization and globalization. In Malaysia, the demographic and epidemiological transition is such that patterns of morbidity and mortality are now similar to those of advanced economies (Western Europe, USA). Cardiovascular disease, cancer and diabetes, mental health problems and accidents and injury are the major causes of burden of disease. These conditions require ongoing medical treatment and appropriate health and welfare programs. The ability of individuals to maximize health and wellbeing following the development of chronic illness and impairment varies as a result of differential access to and the affordability of secondary prevention and treatments. In many cases, individual and community needs have outpaced developments in healthcare provision and expenditure (I).
In recognizing the prevalence of chronic illness and impairment, and the discrimination and stigma that often flow from them, the UN Economic and Social Commission for Asia and the Pacific declared a Decade for Disability in 1992-2001. The second decade (2002-20I I) aims to continue the work to promote inclusive national and state government policies. This initiative has proceeded without a substantial evidence base, and there is still little research data on how people in the region live with chronic diseases and physical and mental impairments. In this paper, we introduce the research we are conducting in Malaysia on this subject, and highlight the importance of an interdisciplinary approach to

\footnotetext{
Correspondence:

Lenore Manderson, PhD FASSA

ARC Federation Fellow and Professor

School of Psychology, Psychiatry and Psychological Medicine

Faculty of Medicine, Nursing and Health Sciences

Monash University, 3800 Australia

Tel: 613-9903 4047

Fax: 613-9903 2501

Email: lenore.manderson@med.monash.edu.au
} 
address the social, economic and medical challenges that derive from this epidemiological transition.

\section{Impairment and Disability in Malaysia}

Malaysia's population is $\mathbf{2 5 . 6}$ million; life expectancy is around 70 for males and 75 for females (2). Most Malaysians can expect to remain independent and productive for a longer period than their parents, but increased longevity brings with it an increased incidence of chronic disease, with attendant problems for individuals, families and communities. Some $50 \%$ of Malaysian men smoke, an important risk factor of numerous cancers, cardiovascular disease and chronic obstructive pulmonary disease; traffic accidents are still common $(2,3)$. Malaysia has escalating rates of hypertension and diabetes mellitus, in part associated with changes in diet and activity: nearly $30 \%$ of the population at the time of the National Health and Morbidity Survey 1997 (the most recent community data available) were obese or overweight and less than $12 \%$ of the population aged 18 years or older had "adequate" physical exercise (2). In addition to impairments following these conditions, approximately $7 \%$ of the Malaysian population are affected by congenital disability or disabilities acquired from accident, injury and disease in childhood and throughout adulthood $(3,4,5)$. An additional $10-15 \%$ of the population are kin or care-givers to this population.

\section{Classifying Function and Disability}

Until recently, people with physical or intellectual impairments were classified using the International Classification of Impairment, Disability and Handicap (ICIDH) (1980). This system of classification emphasised clinical impairments, and while addressing appropriate management strategies for medical rehabilitation teams, it overlooked the social and environmental context and the influence of individual and structural factors on individual outcomes. Consequently, in May 200I, the 54th World Health Assembly adopted the International Classification of Functioning, Disability and Health (ICF) (6).

The revised classification system and its published guidelines provide a framework of disability that acknowledges the different perspectives of health from biological, individual and social perspectives, and provides a tool to describe and compare the health of populations in an international context. The overall aim of the classification system is to provide a unified and standard language and framework to describe health and health-related states. While it describes body functions and structures, it also incorporates broader health-related domains affecting activities and participation such as family structure, economic status, workforce participation and education. The ICF consequently has the potential to identify various disabling and beneficial psychosocial and environmental factors that are relevant in ensuring the full inclusion of persons with impairments. The ICF identifies meaningful sets of related physiological functions, anatomical structures, actions, tasks, and areas of life. The term 'functioning' encompasses all body functions, activities and participation. With the development of the ICF, WHO has moved away from classifying impairment and disability as a 'consequence of disease' classification (i.e., based on the impact of disease) to become a 'components of health' classification (7).

While criticism of this instrumentation continues $(8,9)$, the approach in the ICF is consistent with the contemporary broad-based rehabilitation perspective, with emphasis on the links between health, functioning, and social context. In many respects, the ICF captures the work that has always been a component of medical rehabilitation, including as understood and practised in Malaysia. Rehabilitation medicine is the interdisciplinary management of a person's functioning and health. It aims to restore and maintain body structure and function, minimize activity limitation (impairment/ disability) and restriction in participation (handicap) and prevent further disablement. Depending upon the condition, rehabilitation involves physicians and surgeons, occupational, speech and physiotherapists, nurses, prosthesis and orthotics specialists, psychologists and social workers. Rehabilitation interventions may not change the underlying pathology of a given condition, but can improve a person's symptom control and minimize disability, so enhancing quality of life, improving participation in the community, and decreasing health care costs (7).

Associated personal and environmental factors are critical from a rehabilitation perspective. These have policy and community-wide consequences. For example, providing a wheelchair to someone unable to walk, and training the person to use the wheelchair, is of limited value if curbing and guttering in streets are inadequate, if there are no ramps to allow access to buildings and if public transport is not designed for access by people using wheelchairs. The use of a wheelchair alone also will not change social attitudes or discriminatory and stigmatizing practices that exclude people with physical disabilities from full participation in society and prevent them from maximizing their capabilities in employment, and family and community life. Our understanding of the complex factors that result in disability, however, is limited. In 
particular, the personal factors that facilitate outcomes remain to be identified, and further research is required to enhance our understanding of how different people respond to impairment and disability. Research is also still required to elucidate the social, cultural and economic factors that shape these personal aspects and contribute to the environment of rehabilitation and adaptation, in order to provide better clinical care and to contribute to appropriate policies and programs at a national and local level. Our ongoing research on social aspects of disability and disablement responds to this need.

\section{Resilience: Studying Social Aspects of Impairment and Disability}

As noted, in a clinical context, rehabilitation involves an interdisciplinary team which acknowledges the social and physical environmental factors involved in meeting the needs of individuals who are born with or loose various functions. In research that addresses the broader context, this interdisciplinary approach expands to include social scientists, in order to examine individual and cultural understandings and social and economic consequences of impairment disability. The Resilience Study, the first stages of which we summarize here, was developed in this context. RESILIENCE is an acronym for REsearch into Social Inclusion, Locomotor Impairment and Empowerment through Networking, Collaboration and Education, and is a multi-country, interdisciplinary and collaborative study focusing on living with acquired disability in Australia, Thailand and Malaysia. The study brings together social scientists, public health researchers, clinical medical researchers and practitioners, and includes researcher-consumers, with the aim of generating information of relevance to social policy, rehabilitation medicine and community health.

The Resilience Study is designed to explore the experiences of disability at household and community level. The research focuses on the social context of disability and explores how personal, social, demographic and structural factors moderate or compound the impact of impairment. Drawing on both quantitative and qualitative data using approaches common in medical anthropology (10), the study explores lay understandings and life experiences of people who live with physical difficulties. Their health conditions are often complicated by psychological problems and cognitive difficulties, but also by social isolation and low economic status. Continued disability results in high costs at personal and societal levels in terms of medical and allied healthcare, and in everyday living. There is considerable variation within and between societies and nation states in terms of how care is provided and how the costs of care are met.

The first phase of research focused on local patterns, understandings and life experiences of people who have locomotor impairments for any number of reasons, and the consequent marginalization of individuals and their families. In order to identify the experience of impaired mobility within the community, we adopted a broad definition of impairment, and so included any condition that was considered to affect mobility. These included conditions such as limb amputation from disease, impaired mobility from accident (resulting in spinal cord injury, for example), and loss of function from stroke, but also conditions such as morbid obesity, fractures, arthritis, and asthma which restrict the involvement of the individual in social and economic life.

\section{Quantitative and Qualitative Approaches}

The baseline study was conducted in Selangor in 2003-2004. The quantitative component of the project recruited 210 participants aged 18 years or older. Following stratification into urban and rural areas, a two-stage modified cluster sampling method was employed. Thirty communities were selected randomly, and from health centres in each of these communities, seven individuals were identified as having impaired mobility and were recruited to participate in the study. While some of these participants had access to rehabilitation medicine or community-based rehabilitation services, others had no access to routine services, and no access to physiotherapy or occupational therapy. The quantitative survey used closed questions and scales to gather demographic, socioeconomic and psychometric data, with a structured schedule to collect basic demographic information and socio-economic data. General health and well-being was characterized using the EQ-5D (European Quality of Life Short Form) (II), which includes questions on mobility, self-care, pain and discomfort, ability to undertake usual activities, anxiety and depression, and general health status. In addition, a purpose-specific instrument - Perceived Impact of Problems Profile (PIPP) - was designed and used in the survey to identify self-perceived impact and distress from impaired mobility across a series of domains, including self, relationships, mobility, participation, employment, independence and future. Additional questions were open-ended.

The qualitative component was conducted in a subset of six communities, with a purposively selected population including people who had participated in 
the survey, who elaborated on their experiences of impairment. Other qualitative methods, including interviews with carers and key informants (e.g., health workers, community leaders), group discussions, community mapping and observation provided further contextual information. The qualitative interviews provided participants with an opportunity to discuss at length their individual circumstances, perceptions and experiences, to describe in their own ways and to reflect on their own understandings of the accident or disease, treatment and care, and the changes they experienced personally and socially. The interviews were open-ended; each began with an explanatory statement by the researcher, with each question building on the response of the participant to the prior question. Interviews were taped, checked and reviewed, and tapes were then transcribed for data analysis.

The quantitative data provided us with information on trends and patterns of behaviour, activities and ideas of people with disabilities and their carers. These were explained in depth from the qualitative data. We were not interested primarily in incidence, prevalence or causal links, but rather in identifying common patterns that emerge among people with disabilities. The complementary approach of quantitative and qualitative methods allows us to ensure internal validity, coherence and quality. The approach is inductive, concerned with identifying meaning and patterns rather than designed to test a strict set of hypotheses, and so, gathering "meaning rich" data takes precedence over aggregation and prevalence.

\section{Preliminary Results}

The study sample included 94 men (44.8\%) and I 16 women (55.2\%), ranging in age from 18-90 (60.2 \pm 16.6); men were on average younger than women (57.I \pm I7.5 for men, $62.7 \pm 15.5$ for women). Almost three quarters $(72.4 \%)$ resided in rural areas, and partly reflecting this, mean years of education was low $(5.1 \pm 4.2)$ and the majority $(72.4 \%)$ had primary level education only. Sixty per cent of respondents were married and living with their spouses at the time of the study; $10 \%$ had never married and $29.5 \%$ were separated, divorced or widowed, the latter reflecting the age range of this population. Most $(70.5 \%)$ were Malay and Muslim (71.9\%); 13.8\% were Chinese and $15.2 \%$ Indian with Buddhist, Confucian, and Hindu religious affiliation. $A$ summary of the health status of the respondents in set out in Table I, and illustrates that most $(83 \%)$ experiencing some problems in

Table I. Health profile of study participants by age $(N=210)$

\begin{tabular}{lcccc}
\hline & \multicolumn{4}{c}{ Age Range (Years) } \\
& Total & $\leq \mathbf{5 5}$ & $\mathbf{5 6 - 7 0}$ & $\mathbf{7 I +}$ \\
& $N=210$ & $N-71$ & $N=79$ & $N=59$ \\
& & $(33.8 \%)$ & $(37.6 \%)$ & $(28.6 \%)$ \\
\hline Duration of mobility problems & & & & \\
- Range (years) & $0-60$ & $0-50$ & $0-60$ & $1-25$ \\
- Mean years \& SD & $6.8 \pm 10.9$ & $10.4 \pm 14.5$ & $5.4 \pm 9.8$ & $4.5 \pm 4.3$ \\
Cause of mobility problems (\%)' & & & & \\
- Sickness/lllness & 61.0 & 45.1 & 65.8 & 73.3 \\
- Accident & 23.8 & 43.7 & 19.0 & 6.7 \\
- Since birth & 2.4 & 4.2 & 2.5 & 0.0 \\
- Don't know & 11.9 & 8.5 & 13.9 & 13.3 \\
- Others & 9.0 & 8.5 & 5.1 & 15.0 \\
Other health problems (\%)' & & & & \\
- Hypertension & 43.8 & 23.9 & 59.0 & 47.5 \\
- Diabetes & 35.4 & 26.8 & 44.3 & 33.9 \\
- Arthritis & 29.0 & 16.8 & 36.7 & 33.3 \\
- Stroke & 18.2 & 7.0 & 25.3 & 22.0 \\
- Heart disease & 14.8 & 7.0 & 12.7 & 26.7 \\
- Lung disease (including asthma) & 8.7 & 2.8 & 11.5 & 11.8 \\
- Tuberculosis & 2.9 & 1.4 & 5.1 & 1.7 \\
- Others & 12.1 & 15.5 & 9.1 & 11.9 \\
\hline
\end{tabular}

\footnotetext{
'Participants reported more than one health problem
} 
walking, with $9 \%$ confined to bed. Only a small proportion of the sample $(8 \%)$ was able to walk about without problems, for example, because they felt confident using a gait aid. More than half $(62 \%)$ of the sample perceived that they had no problems with self care, and one third $(31 \%)$ felt that they had some problems in this respect. The remaining $7 \%$ of the sample were not able to wash or dress themselves. More than half of the sample (53\%) felt that they could perform their "usual" activities, including working or doing housework or family activities, but with some problems. While $26 \%$ of the respondents did not have problems with performing their usual activities, $21 \%$ reported that such activities were impossible to perform.

More than half of the respondents $(63 \%)$ reported experiencing moderate pain or discomfort at the time of interview, and more than one quarter $(27 \%)$ experienced extreme pain or discomfort. Only a small proportion (10\%) was free of pain or discomfort. While nearly one quarter of the respondents (23\%) reported no feelings of anxiety or depression, $46 \%$ and $31 \%$ respectively were moderately or extremely anxious or depressed. When asked about their feelings regarding general health (using the scale of $0-100$ ), the single most frequent response was the mid-point of $50($ mean $=60)$.

As described, the PIPP instrument collected data across a number of domains. The major differences were that there was a greater impact on relational items (confidence, mood, satisfaction) and on the ability to have a close relationship for younger people than older respondents. There was also a trend towards greater impact in the emotion items for urban participants compared with rural participants in terms of overall satisfaction with life, moods and feelings, and sense of confidence, and greater distress on the relational items for younger people in terms of ability to relate to people in authority, ability to relate to neighbours and friends and ability to relate to relatives. Interview data with health workers emphasized that these people often lacked social support to allow them to interact with others and participate in society, and in consequence the social exclusion is one reason for people's reported distress and depression.

The qualitative data allows for some interpretation of these findings. People drew attention to the impact of loss of mobility on their mental health and on their independence and autonomy. One man, for example, recalled that he was very depressed: "I used to walk and live an independent life, then suddenly I can't do everything. I realized it's fate but what can I do? I just have to be really patient and leave it up to God." Many participants drew on faith-based ideas - that their health condition was in God's hands, for example, or that their health condition was the result of fate. Older participants reflected on the inevitability of aging, and drew solace from the fact that they had already lived a full life. Participants also reflected on the difficulty of being as independent as possible, rejecting family support that they felt was inappropriate and demeaning. Hence the comment, "I feel annoyed most of the time when people come to me and try to do things for me. Let me do it myself. If I can't, then l'll ask for help."

While people aspired to be as independent as possible, data from health workers and community members indicate that there is considerable misunderstanding about the capability of individuals with limited mobility. One health worker, for example, reflected that "if they (people with limited mobility) do have support, it is only partial ... and they have low self-esteem and low self-confidence." The assumption that such people have "low self-confidence", in turn influences how others in the community interact with them. Thus a village headman explained that "we invite all residents including the disabled, whenever we conduct any program for the village, but whether they want to join in or not is their own decision. We never force them to be involved. Maybe because some of them have low self-esteem, although we never treat them differently." Yet at the same time, this participant and others acknowledged other practical barriers to social participation and inclusion, such as lack of an attendant carer, transportation problems, financial difficulties and environmental barriers.

\section{Conclusion}

The Malaysian Government is faced with numerous challenges to current health policies and systems that derive from changes in standards of living, changing disease patterns (from communicable to noncommunicable diseases) and demographic changes. The increased incidence in chronic and degenerative diseases related to changes in lifestyle and behaviour, for example, highlights the importance for changes to occur in the mix, integration and delivery of health services, and changes in public health and prevention. The need for review and, as necessary, reform of medical and health services has arisen concurrently with increased commitment to ensuring equity and maximizing the social participation and capabilities of all citizens. The former Ministry of National Unity and Social Development (now the Ministry of Women, 
Family and Community Development) presented a draft Persons with Disabilities Act for discussion in parliament in 2002. This Act provides the legislative means for strategies and programs to ensure such participation and inclusion. Improved and appropriate social and medical strategies are required to ensure that Malaysians with chronic illness or disability enjoy productive lives and maximize their capabilities. The consequent changes in policies, programs and strategies to ensure universal access to appropriate health care and services, and to ensure the inclusion of people with impairments, require a strong evidence base. This points to the need for continued research regarding social as well as medical aspects of chronic disease, impairment and disability. Our research on social aspects of impairment is a first step towards this end.

\section{Acknowledgement}

The baseline research was facilitated by the Australian Research Council (ARC) Federation Fellowship Grant (Manderson, No. FF024I243), an ARC Discovery Grant (Manderson, Bennett and Disler DP04496/4, The University of Melbourne), and an Intensified Research Priority Area (IRPA) Grant No. 06-02-03-0534. The research teams from all three countries met as a consortium in June 2003 in Khon Kaen (northeast Thailand) and in May 2004 in Vientiane, Lao PDR to share data methods, instruments and analysis. Ethics approval for the research in Malaysia was provided by the University of Malaya Medical Centre, Medical Ethics Committee (Ethics No. 344.I2). Team members involved in various ways in the development of instruments, data collection and data management and analysis are Akmal Hafizah, Kumaran s/o Ramakrishnan, Yi Lin, Noor Ezawanee Badaruddin, Kavitha Uma, Maslina Yazid, Shaari Mohd Yatim, Elizabeth Bennett, Julie Pallant, Sari Andajani-Sutjahjo and Cate Bailey.

\section{References}

I. Mathers CD, Sadana R, Salomon JA, et al. Healthy life expectancy in 191 countries, 1999. Lancet 2000; 357: |685-169|.

2. Hussein RHT, Anuar H. Report of the Second National Health and Morbidity Survey Conference, Hospital Kuala Lumpur, 20-22 November 1997. Kuala Lumpur: Public Health Institute, Ministry of Health, Malaysia 1997.

3. Karim HA. The elderly in Malaysia: demographic trends. Med J Malaysia I997; 52, 3: 206-2I 2.

4. Mustaffa BE. Diabetes epidemic in Malaysia. Med J Malaysia 2004; 59, 3: 295-296.

5. Sherina MS, Rampal L, Mustaqim A. The prevalence of depression among the elderly in Sepang, Selangor. Med J Malaysia 2004; 59, 3: 45-49.

6. WHO (World Health Organization) International Classification of Functioning, Disability and Health: ICF. Geneva: WHO, 200I.

7. Stucki G, Ewert T, Cieza A. Value and application of the ICF in rehabilitation medicine. Disabil Rehabil 2002; 24, 17: 932-938.

8. Imrie R. Demystifying disability: a review of the International Classification of Functioning, Disability and Health. Sociol Health IIIn 2004; 26, 3: 287-305.

9. Schuntermann MF. The implementation of the international classification of functioning, disability and health in Germany: experiences and problems. Int J Rehabil Res 2005; 28, 2: 93-102.

10. Manderson L. Gender, normality and the post-surgical body. Anthropology and Medicine 1999; 6(3): 38I-394.

II. Brooks R. EuroQoL: the current state of play. Health Policy 1996; 37, I: 53-72. 